UDC 811.131.1'367.625811.163.41'367.625

811.131.1:811.163.41

DOI 10.18485/italbg.2016.2.4

https://doi.org/10.18485/italbg.2016.2.4

Jelena Puhar*

Università di Belgrado

\title{
VARIAZIONE PRIMARIA DI 'PORTARE’ E I SUOI TRADUCENTI SERBI IN IMAGACT
}

\begin{abstract}
I verbi più frequenti in tutte le lingue parlate sono i verbi di azione, i quali denotano azioni fisiche compiute dall'agente (Mrazović 2009: 69). Come riporta Yi (2015: 8), molti verbi di azione sono verbi generali, caratterizzati da forte ambiguità e spesso non vicendevolmente traducibili su tutte le azioni a cui si estendono nelle diverse lingue, per cui trovare il verbo di azione adeguato in una lingua straniera può creare problemi. Pertanto, lo scopo del presente lavoro è quello di dare un contributo alle ricerche sulla semantica del verbo generale 'portare' e i suoi traducenti serbi, riportando i risultati derivati da IMAGACT, un'ontologia interlinguistica di tipi azionali dei verbi di azione rappresentati attraverso immagini in movimento. Verranno esaminati, dal punto di vista contrastivo, diversi tipi di azione espressi dal verbo 'portare' i e suoi traducenti serbi in IMAGACT, prestando particolare attenzione alle differenze tra le due lingue dovute anche all'aspetto verbale grammaticalizzato in serbo.
\end{abstract}

Parole chiave: verbi di azione, tipi azionali, IMAGACT, italiano, serbo, semantica.

\section{INTRODUZIONE}

Lepschy (2007: 70) afferma che "ogni lingua impone ai suoi parlanti un modo diverso di vedere il mondo, e inevitabilmente li costringe a esprimersi secondo categorie concettuali e strutture logiche diverse da lingua a lingua". Questa affermazione riguarda anche i verbi di azione in quanto le diverse lingue categorizzano l'universo delle azioni in maniera particolare (Gagliardi 2014: 29). Molti verbi di azione sono polisemici e causano problemi nella traduzione. Le risorse semantiche attuali, quali WordNet (Fellbaum 1998), FrameNet (Baker et al. 1998), VerbNet (Kipper Schuler 2005) non offrono una disambiguazione chiara dei sensi di parola (Yi 2015: 25). Questa lacuna viene colmata, almeno per i verbi di azione, dal progetto

\footnotetext{
*jelenapuhar@yahoo.com
} 
IMAGACT $^{1}$, realizzato da LABLITA di Firenze, il Dipartimento di Scienze della Comunicazione dell'Università di Siena e l'Istituto di Linguistica Computazionale "Antonio Zampolli" (Gagliardi 2014: 29). Come obiettivo principale del progetto, destinato sia agli apprendenti di una lingua straniera che agli studiosi di lingue, si propone la creazione di un'ontologia interlinguistica accessibile online, nella quale sono identificati i tipi di azione dei verbi azionali in diverse lingue.

IMAGACT, il quale si focalizza su verbi di azione estratti dai corpora dell'italiano parlato (LABLITA, LIP, CLIPS) e dal corpus di inglese parlato $\mathrm{BNC}^{2}$, contiene circa 500 verbi di azione più frequenti presenti nei corpora di ciascuna lingua. I tipi di azione identificati nei corpora sono raffigurati mediante le scene registrate in studio oppure realizzate come animazioni in 3D. Visto che i tipi azionali rappresentati dalle scene sono indipendenti dalla lingua, IMAGACT è potenzialmente estensibile a tutte le lingue. A tal fine è stata creata l'infrastruttura IMAGACT4ALL. Il lavoro di estensione di competence-based a nuove lingue viene eseguito da annotatori madrelingua, i quali, accedendo alla parte di IMAGACT dedicata all'estensione competence-based, per ogni tipo azionale attribuiscono uno o più lemmi che meglio lo rappresentano nella loro lingua (Yi 2015: 61). Il lemma viene annotato all'infinito e le scene vengono descritte con le frasi attive. In IMAGACT viene considerata solo la variazione primaria dei verbi, cioè soltanto il loro senso proprio, limitato alle azioni fisiche, mentre il senso traslato viene escluso. La rappresentazione delle azioni attraverso le scene permette la comprensione del loro valore semantico indipendentemente dalla lingua e consente l'estensibilità dell'ontologia a diverse lingue, tra le quali anche alla lingua serba. L'utente di IMAGACT può verificare come si identificano i tipi azionali nella lingua che gli interessa nonché paragonare l'ambito di applicazione di due verbi di lingue diverse.

In questo contributo ci proponiamo di mettere in luce i risultati derivanti dall'analisi contrastiva dei tipi azionali del verbo generale 'portare' e i suoi traducenti serbi in IMAGACT. Riteniamo la variazione verticale di 'portare' particolarmente interessante per l'analisi contrastiva tra le due lingue in quanto 'portare' copre campi di variazione molto più ampi rispetto al verbo nositi con il quale 'portare' è comunemente tradotto in dizionari bilingui. Perciò lo scopo di questo lavoro è appunto quello di esaminare i verbi generali serbi (e i loro prefissati) corrispondenti ai tipi azionali di

${ }^{1}$ Nel suo primo rilascio l'interfaccia IMAGACT, sul sito http://www.imagact.it, era disponibile per la lingua italiana, inglese, spagnola, cinese e danese. Sul sito http://62.48.36.230:8180/imagact/query/dictionary.seam è disponibile una demo dell'estensione dell'ontologia alla lingua tedesca, hindi, polacca, portoghese, urdu e serba.

${ }^{2}$ British National Corpus. 
'portare' in IMAGACT, prestando particolare attenzione ai criteri della loro selezione. Inoltre, la nostra attenzione si focalizzerà sull'aspetto verbale grammaticalizzato in serbo in quanto l'aspetto verbale è presente nella struttura semantica di ogni verbo serbo.

La prima parte del nostro lavoro sintetizza i modi principali della classificazione dei verbi nelle grammatiche italiane nonché la classificazione dei verbi di azione presenti in IMAGACT, proposta da Moneglia (2010). Dato che i verbi serbi, oltre ad essere classificabili secondo gli stessi criteri come i verbi italiani, vengono distinti anche in base al loro aspetto verbale, nella seconda parte ci focalizziamo appunto sulle caratteristiche dell'aspetto verbale serbo, evidenziando la suddivisione dei verbi serbi in quelli perfettivi, imperfettivi e biaspettuali. Nella parte centrale esaminiamo la variazione verticale di 'portare' in IMAGACT, prestando particolare attenzione ai verbi generali serbi e ai loro prefissati i quali equivalgono ai tipi azionali di 'portare'. Infine, ci concentriamo sulla variazione orizzontale di 'portare' e dei suoi traducenti serbi allo scopo di dimostrare che i tipi azionali possono essere presi in considerazione a un livello ontologico indipendente dal linguaggio, come ipotizzato da Moneglia (2011: 3). Anche la nostra analisi confermerà che i verbi generali sono soltanto parzialmente equivalenti in diverse lingue, nel nostro caso in italiano e serbo, in quanto, come nota Ježek (2011: 26), "le lingue divergono sia nel mondo in cui segmentano i concetti, sia nel modo in cui associano uno stesso contenuto agli elementi lessicali".

\section{CLASSIFICAZIONE DEI VERBI DI AZIONE IN IMAGACT}

Secondo Ježek (2011: 116, 117) i verbi sono classificabili secondo tre criteri principali. In base all'Aktionsart, i verbi si distinguono in verbi stativi, continuativi, trasformativi, risultativi, puntuali (cfr. Bertinetti 2011: 26-33). In base al concetto di valenza, i verbi vengono classificati in verbi zerovalenti, monovalenti, bivalenti, trivalenti, tetravalenti (cfr. D'Achille 2003: 170; Graffi 2012: 53, 54; Ferrari \& Zampese 2016: 118). Il terzo modo della classificazione si basa sul significato denotativo del verbo. In base a

questo criterio verbi possono essere distinti in verbi di moto, di maniera, di percezione, di misura, di lancio ecc. (Ježek 2011: 117). Inoltre, Salvi (2013: 91), in base alla realizzazione interna o esterna del soggetto, suddivide $\mathrm{i}$ verbi in quelli innacusativi e accusativi.

L'autore di IMAGACT, Massimo Moneglia (2010), in base alle proprietà semantiche e pragmatiche dei verbi di azione presenti nel corpus di IMAGACT, propone la loro suddivisione in verbi di attività, verbi di movimento 
e verbi generali. I verbi di attività riguardano un tipo cognitivo di azione (Moneglia 2010: 1-5). Se prendiamo in considerazione, ad esempio, il verbo 'bere' e le scene che in IMAGACT lo raffigurano, possiamo concludere che, oggettivamente, le azioni che si eseguono presentano delle differenze. In 1a) la persona sorbisce un liquido con la cannuccia, in 1b) il gatto prende l'acqua che sgorga dal rubinetto, in 1c) la persona inghiottisce un liquido prendendolo da un recipiente. È facilmente osservabile che in 1a) l'agente compie l'azione stringendo le labbra, in $1 \mathrm{~b}$ ) nella realizzazione dell'azione viene implicata la lingua, mentre in 1c) l'azione del bere viene eseguita senza usare le labbra e la lingua. Nonostante queste differenze, linguisticamente e concettualmente, viene realizzata la stessa azione descrivibile con il verbo italiano 'bere' e con il suo equivalente serbo piti.

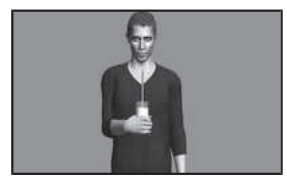

1a)

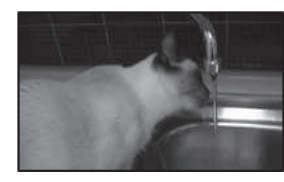

1b)

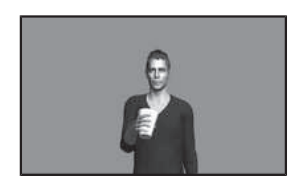

1c)

Alla seconda sottoclasse dei verbi azionali appartengono i verbi di movimento, i quali indicano il cambiamento di posizione di un'entità nello spazio (Strudsholm 2011: 1). Infine, i verbi generali, i quali designano le azioni quotidiane più frequenti, non riguardano solo un'azione, ma sono estensibili ad azioni diverse. Per esempio, il verbo 'lasciare' si riferisce a tipi azionali diversi, $\mathrm{i}$ quali in serbo sono individuati da più predicati. Il verbo ostaviti corrisponde a 'lasciare' nei campi di applicazione di 'lasciare' equivalenti al verbo 'dare', come nella scena descritta in IMAGACT con le frasi 'Marta lascia / dà la borsa a Mario', alle quali corrispondono le frasi serbe Marina ostavlja / je ostavila / daje / je dala torbu Mirku. Il verbo serbo ostaviti però non è applicabile a 'lasciare' alle variazioni in cui 'lasciare' equivale a 'lasciar cadere'. In questo caso il serbo richiede il verbo ispuštati 'lasciar cadere', come nell'esempio 'Marta lascia / lascia cadere la tazza': Marina ispušta / je ispustila šolju. Inoltre, il verbo serbo ostaviti non si applica ai campi in cui 'lasciare' è localmente equivalente al verbo 'allentare'. Il verbo serbo corrispondente in questo caso è popuštati 'allentare'.

Secondo Moneglia (2011) i verbi generali rappresentano i verbi meno predicibili per le opere lessicografiche disponibili in quanto i loro significati risultano ambigui, come verrà dimostrato anche in questa sede, nella parte dedicata alla variazione primaria del verbo 'portare'. 


\section{ASPETTO VERBALE IN SERBO}

Visto che, come accennato in precedenza, ogni verbo serbo possiede l'aspetto nella struttura semantica del verbo, prima di esaminare i tipi azionali di 'portare', ci soffermeremo sull'aspetto verbale grammaticalizzato in serbo. Belić (2000: 195) per l'aspetto verbale sottintende la categoria grammaticale che indica maniera in cui è concepito lo svolgimento dell'azione espressa dal verbo. Tomelleri (2008: 11) aggiunge che l'aspetto verbale in lingue slave è 'l' espressione formale di perfettivo e imperfettivo mediante affissi". La maggior parte dei verbi in serbo ha la coppia aspettuale, una coppia di due verbi di aspetto perfettivo e imperfettivo, i quali si distinguono tra di loro per la semantica grammaticale dell'aspetto e per la presenza del suffisso o del prefisso esprimenti questa differenza (Lentovskaya 2007: 2). Il verbo di aspetto imperfettivo esprime un'azione in corso, mentre i verbi perfettivi denotano azioni concluse (Klajn 2007: 104). A molti verbi imperfettivi corrisponde più di un verbo perfettivo; ad esempio dal verbo pisati mediante la prefissazione derivano i verbi perfettivi napisati e raspisati. ${ }^{3}$ Il serbo possiede anche verbi biaspettuali, i quali con un lemma designano ambedue gli aspetti. Il loro significato aspettuale concreto deriva dal contesto (Stanojčić 2010: 161). Considerato che l'utilizzo del verbo di aspetto perfettivo o di aspetto imperfettivo ha conseguenze anche sul piano semantico del verbo, il lemma perfettivo e quello imperfettivo hanno la propria entrata lessicale sui dizionari, per cui i traducenti serbi di 'portare' in IMAGACT nel paragrafo 5 sono sistematizzati nella tabella (Tabella 1) sia in base ai tipi azionali che in base all'aspetto verbale.

\section{VARIAZIONE VERTICALE DI 'PORTARE' E I SUOI TRADUCENTI SERBI}

Dal punto di vista semantico, la maggior parte dei verbi in IMAGACT sono verbi generali perché si estendono a più tipi di azioni diversi. La classe semantica dei verbi generali parte dal filosofo Ludwig Wittgenstein (1953) e dal suo concetto di generalità estensionale. Wittgenstein (in Panunzi \& Moneglia 2003: 6) individua il concetto di "somiglianza di famiglia come sistema di relazioni che legano tra loro oggetti (in questo caso eventi) denotati da uno stesso predicato, ma diversi l'uno dall'altro". Le diverse "famiglie" rappresentano i tipi di azione che fanno parte dell'estensione del predicato (Panunzi \& Moneglia 2003: 6). Tali tipi di azione vengono nominati variazione verticale del verbo (Moneglia et al. 2012: 409). Ba-

\footnotetext{
${ }^{3}$ Il prefisso $n a$ - cambia solo l'aspetto del verbo, mentre il prefisso raz- ne modifica anche il significato lessicale (raspisati 'indire').
} 
sandosi sui tipi azionali del verbo 'portare' derivati da IMAGACT, nei seguenti paragrafi (4.1-4.8) esamineremo i traducenti serbi di ogni campo di variazione verticale di 'portare'.

Da IMAGACT risulta che il verbo 'portare' possiede otto tipi azionali: a) possesso continuo, b) transizione oggettiva benefattiva, c) transizione oggettiva locativa, d) dislocazione dell'oggetto, e) accompagnamento al movimento dell'oggetto, f) transizione oggettiva locativa con un veicolo come oggetto tematico, g) trasporto dell'oggetto, h) trasporto dell'oggetto con uno strumento come soggetto.

\subsection{Possesso continuo}

Il primo tipo azionale in IMAGACT è rappresentato con la frase 'Maria porta la valigia' a cui corrisponde la frase serba Marina nosi kofer. In questa istanza la destinazione dell'azione non viene espressa, ma viene focalizzato il fatto che l'agente tiene in mano il tema durante la transizione. Alla scena raffigurante la frase riportata è applicabile il verbo serbo nositi, con il quale 'portare' è comunemente tradotto in dizionari bilingui.

\subsection{Transizione oggettiva benefattiva}

Il tipo azionale designante la transizione oggettiva benefattiva in IMAGACT è indicato con la frase 'Fabio porta il libro a Maria', il cui corrispondente serbo sono le frasi Mirko odnosi / je odneo / donosi / je doneo knjigu Marini. In questa variazione, oltre al processo di cambiamento spaziale dell'oggetto, viene espresso anche il beneficiario. Il verbo 'portare' è equivalente a 'dare' ed è traducibile in serbo con il verbo nositi e i suoi prefissati imperfettivi donositi e odnositi. I prefissi $d o$ - e od-aggiungono il significato lessicale al verbo senza modificarne l'aspetto (Klajn 2007: 109). Il prefisso do- indica la direzione dell'azione verso il raggiungimento di una meta, mentre il prefisso od- ha significato ablativo. Nella scena descritta con la frase sopra indicata, l'agente raggiunge il beneficiario, che a livello superficiale, rappresenta la sua destinazione. Perciò la scena è descrivibile anche con i verbi perfettivi serbi doneti e odneti. ${ }^{4}$

\subsection{Transizione oggettiva locativa}

Alla variazione riguardante la transizione oggettiva locativa vengono attribuiti gli stessi traducenti serbi come nel tipo azionale precedentemente analizzato. Ciò che differenzia questo tipo azionale da quello sopra riportato

${ }^{4}$ Il loro tema -neti deriva dal verbo nesti, una forma antica dell'infinito di nositi (Stakić 2010: 145). 
è l'indicazione, in modo esplicito, della destinazione dello spostamento dell'oggetto inanimato, come si può individuare nell'esempio 'Fabio porta la sedia al tavolo': Mirko donosi / odnosi / je doneo / je odneo stolicu do stola. La presente variazione in IMAGACT è raffigurata anche dalla scena descritta con la frase 'Fabio porta il radiatore fuori', alla quale equivalgono le frasi serbe Mirko prenosi / iznosi / nosi / odnosi radijator. Oltre ai verbi nositi e odnositi, il verbo sintagmatico 'portare fuori' è traducibile anche con il verbo iznositi, il cui prefisso $i z$ - indica la separazione dalla parte interna verso la parte esterna, e con il verbo prenositi, indicante lo spostamento da un luogo.

\subsection{Dislocazione dell'oggetto}

Il quarto tipo azionale, la dislocazione dell'oggetto, è rappresentato con le frasi 'Maria porta / piglia / si porta via / prende con sé una valigia' a cui corrisponde Marina uzima / je uzela / odnosi / je odnela / je ponela $k o f e r$. Il focus è sul fatto che l'agente prende con sé il tema. Dunque, i verbi applicabili alla presente variazione hanno valore ablativo. Il traducente serbo è la coppia aspettuale odnositi-odneti prefissata con il prefisso od-indicante un allontanamento. I verbi odnositi e odneti in questo caso sono localmente equivalenti ai verbi uzimati e uzeti 'prendere'. Oltre ai verbi serbi aventi il significato ablativo, in questa istanza 'portare' è traducibile anche con il verbo serbo poneti 'portare con sé'.

\subsection{Accompagnamento al movimento dell'oggetto}

Per l'istanza che designa l'accompagnamento al movimento dell'oggetto in IMAGACT sono riscontrabili i seguenti esempi:

1) I poliziotti portano / conducono / accompagnano il ladro in prigione.

Policajci vode / sprovode / dovode lopova.

2) Fabio porta / conduce / accompagna / guida il cieco dall'altra parte della strada.

Mirko vodi / prevodi / je preveo slepog čoveka preko ulice.

3) Fabio porta / tira il cavallo verso la stalla. Mirko vodi konja / vuče konja na kanapu.

4) Il pilota porta i passeggeri a destinazione. Mirko vozi Marinu u helikopteru.

Dall'analisi degli esempi forniti, risulta che la variazione di nositi non corrisponde ai campi in cui 'portare' equivale ai verbi 'accompagnare', 'condurre' e 'guidare'. Come si può facilmente osservare, in questo tipo 
azionale l'oggetto è animato. Nelle frasi 1), 2), 3) 'portare' è traducibile con il verbo generale serbo voditi e i suoi prefissati dovoditi e sprovoditi (in 1) e prevoditi (in 2). I prefissi dei verbi riportati sono lessicali in quanto aggiungono valore semantico alla base. Il prefisso do- denota l'azione eseguita allo scopo di raggiungere una meta, il prefisso pre-indica lo spostamento da un posto ad un altro. In 4) il significato di 'portare' si estende al significato del verbo 'trasportare'. In questo caso il serbo richiede il verbo voziti, il quale esprime l'azione di spostamento di un oggetto mediante un autoveicolo. Visto che le scene raffigurano il raggiungimento della destinazione, sono descrivibili anche con i verbi perfettivi dovesti, sprovesti e prevesti. ${ }^{5}$

\subsection{Transizione oggettiva locativa con un veicolo \\ come oggetto tematico}

La variazione relativa alla transizione oggettiva locativa (con un veicolo come oggetto tematico) si differenzia dal terzo tipo azionale soltanto per il tema, il quale, essendo un veicolo, non può essere tenuto in mano. I traducenti serbi sono le coppie aspettuali uvoziti-uvesti, uterivati-uterati, uparkiravati-uparkirati. Inoltre, alla presente variazione si può applicare il verbo biaspettuale parkirati, il quale, per descrivere questa scena, può essere usato sia in forma imperfettiva che in quella perfettiva in quanto nella scena indicata dalla frase 'Fabio porta la macchina in garage', il cui corrispondente serbo sono le frasi Mirko uteruje / je uterao / uvozi / je uvezao / uparkirava /je uparkirao / je parkirao kola u garažu prima viene rappresentata l'azione in corso e poi viene raffigurata anche la sua conclusione.

\subsection{Trasporto dell'oggetto}

Al tipo azionale che designa il trasporto dell'oggetto sono attribuite le frasi 'L'autotrasportatore porta / trasporta il carico con il camion': Kamion prevozi teret. In questa istanza la relazione viene instaurata fra il tema e l'agente che trasporta l'oggetto non animato utilizzando un veicolo. Il verbo serbo corrispondente a questo tipo d'azione è prevoziti indicante il trasferimento di un oggetto da un posto ad un altro mediante un mezzo di trasporto. Come si può osservare dall'esempio, in questa istanza 'portare' è localmente equivalente al verbo 'trasportare'.

${ }^{5}$ I verbi voditi e voziti per la perfettizzazione usano il tema -vesti ritrovabile soltanto nei composti (Piper \& Klajn 2013: 178). 


\subsection{Trasporto dell'oggetto con uno strumento \\ come soggetto}

Con le frasi 'Il nastro trasportatore porta / trasporta i mattoni', a cui equivale la frase serba Pokretna traka prenosi cigle, è descritta la scena indicante la variazione che denota il trasporto dell'oggetto, ma il soggetto della presente frase è uno strumento. Il lemma serbo annotato a questa variazione è soltanto il lemma imperfettivo prenositi in quanto nella scena non è rappresentata la conclusione dell'azione. Il prefisso pre-, indicante lo spostamento dell'oggetto da un posto ad un altro, è lessicale perché non modifica l'aspetto del verbo, ma solo aggiunge il valore semantico alla base verbale.

\section{RISULTATI DELL'ANALISI}

I risultati derivati dalle due analisi parallele condotte sull'italiano e il serbo confermano che il verbo 'portare' indica più azioni diverse. Dagli esempi riportati emerge che i campi di variazione del verbo 'portare' sono assai più ampi rispetto al verbo serbo nositi. Di seguito riportiamo una tabella riassuntiva con i lemmi presenti nel corpus come traducenti serbi di 'portare' nelle variazioni menzionate nonché alcune delle immagini raffiguranti le scene in IMAGACT. 
Tabella 1. Variazione verticale di 'portare'e $i$ suoi traducenti serbi

\begin{tabular}{|c|c|c|c|c|}
\hline $\begin{array}{l}\text { Tipo di } \\
\text { azione }\end{array}$ & Immagine & $\begin{array}{l}\text { Verbo serbo } \\
\text { imperfettivo }\end{array}$ & $\begin{array}{c}\text { Verbo serbo } \\
\text { perfettivo }\end{array}$ & $\begin{array}{l}\text { Verbo serbo } \\
\text { biaspettuale }\end{array}$ \\
\hline $\begin{array}{l}\text { Possesso } \\
\text { continuo }\end{array}$ & & nositi & & \\
\hline $\begin{array}{l}\text { Transizione } \\
\text { oggettuale }\end{array}$ & & $\begin{array}{l}\text { donositi } \\
\text { odnositi }\end{array}$ & $\begin{array}{l}\text { doneti } \\
\text { odneti }\end{array}$ & \\
\hline $\begin{array}{l}\text { Transizione } \\
\text { oggettuale } \\
\text { locativa }\end{array}$ & & $\begin{array}{c}\text { donositi } 1^{6} \\
\text { odnositi } 1 \\
\text { nositi } 2 \\
\text { iznositi } 2 \\
\text { prenositi } 2\end{array}$ & $\begin{array}{l}\text { doneti } 1 \\
\text { odneti } 1\end{array}$ & \\
\hline $\begin{array}{l}\text { Dislocazione } \\
\text { dell'oggetto }\end{array}$ & & $\begin{array}{l}\text { odnositi } \\
\text { uzimati }\end{array}$ & $\begin{array}{c}\text { odneti } \\
\text { uzeti } \\
\text { poneti }\end{array}$ & \\
\hline
\end{tabular}

${ }^{6}$ Con 1 è indicato il verbo corrispondente all'immagine superiore e con 2 a quella inferiore. 


\begin{tabular}{|c|c|c|c|c|}
\hline $\begin{array}{l}\text { Accompa- } \\
\text { gnamento al } \\
\text { movimento } \\
\text { dell'oggetto }\end{array}$ & 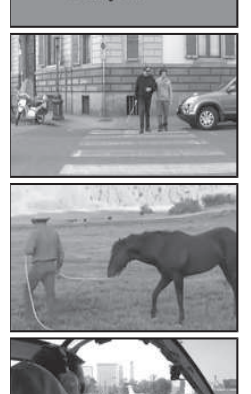 & $\begin{array}{c}\text { voditi } 1,2,3 \\
\text { dovoditi } 1 \\
\text { sprovoditi } 1 \\
\text { prevodi } 2 \\
\text { vući } 3 \\
\text { prevoziti } 4 \\
\text { dovoziti } \\
\text { pratiti } \\
\text { šetati }\end{array}$ & $\begin{array}{c}\text { prevesti } 2 \\
\text { dovesti } \\
\text { dopratiti } \\
\text { ispratiti }\end{array}$ & \\
\hline $\begin{array}{l}\text { Transizione } \\
\text { oggettuale } \\
\text { locativa } \\
\text { (il tema è } \\
\text { un veicolo) }\end{array}$ & $\frac{1}{0} 2$ & $\begin{array}{c}\text { uvoziti } \\
\text { uterivati } \\
\text { uparkiravati }\end{array}$ & $\begin{array}{c}\text { uvesti } \\
\text { uterati } \\
\text { uparkirati }\end{array}$ & parkirati \\
\hline $\begin{array}{l}\text { Trasporto } \\
\text { dell'oggetto }\end{array}$ & & prevoziti & & \\
\hline $\begin{array}{l}\text { Trasporto } \\
\text { dell'oggetto } \\
\text { (il soggetto } \\
\text { è uno } \\
\text { strumento) }\end{array}$ & tit & prenositi & & \\
\hline
\end{tabular}

Dalla tabella emerge che i traducenti serbi dei tipi azionali del verbo 'portare' possono essere i verbi generali serbi nositi, voditi e voziti (e i loro derivati prefissali), i cui campi di variazione sono più ridotti rispetto a 'portare', come è facilmente osservabile in IMAGACT nella funzione Compare. Utilizzando questa funzione, l'utente può confrontare l'uso del 
verbo portare con i suoi traducenti serbi osservando le scene rappresentanti ciascun tipo azionale.

Inoltre, i dati riportati nella tabella permettono di individuare ulteriori informazioni oltre a quelle presentate finora. Il traducente serbo del verbo 'portare' può essere anche la coppia aspettuale pratiti-ispratiti 'accompagnare' nel tipo azionale accompagnamento al movimento dell'oggetto rappresentato in IMAGACT dalla scena descritta con le frasi 'Fabio porta Marta alla stazione ferroviaria in macchina' e 'Fabio accompagna Marta alla stazione ferriovaria' i cui corrispondenti serbi sono le frasi Mirko dovozi / je dovezao / prati / je ispratio Marinu na železničku stanicu. Notiamo inoltre che i verbi serbi šetati 'portare a spasso' e vući 'tirare' sono applicabili al tipo azionale in cui 'portare' equivale a 'condurre' quando a essere condotti sono gli animali, come nelle scene descritte con le frasi 'Fabio porta il cane a spasso' e 'Fabio porta / tira il cavallo verso la stalla', alle quali corrispondono le frasi serbe Mirko vodi psa u šetnju / šeta psa e Mirko vodi konja / vuče konja na kanapu.

Per quanto riguarda l'aspetto verbale serbo, dalla tabella emerge che, dal punto di vista dell'aspetto verbale, i traducenti serbi sono: a) i verbi aventi la coppia aspettuale (ad es. dovoditi-dovesti), b) i verbi imperfettivi senza correlativo aspettuale (ad es. prenositi), c) il verbo biaspettuale (parkirati). Come si evince dalla tabella, al momento in IMAGACT non sono disponibili le scene che rappresentano tutti i membri delle coppie aspettuali per ogni verbo serbo in quanto il progetto IMAGACT si basa sui verbi estratti dai corpora delle lingue non aventi l'aspetto verbale grammaticalizzato. Tuttavia, visto che l'imperfettività e la perfettività in serbo (ma anche in altre lingue slave) sono determinanti per la selezione del verbo di azione adatto, sarebbe opportuno arricchire IMAGACT con le scene descrivibili con i verbi perfettivi mancanti, rendendolo in questo modo ancora più utile per i suoi utenti.

\section{VARIAZIONE ORIZZONTALE DI 'PORTARE' E I SUOI TRADUCENTI SERBI}

Oltre alla variazione verticale, i verbi generali possiedono la variazione orizzontale, la quale si verifica all'interno di ogni tipo d'azione (Moneglia \& Panunzi 2010: 28). Moneglia (2005: 4; 2011: 2) nota che la produttività dei tipi di azioni diversi è identificabile empiricamente mediante la verificazione della produttività dell'azione. Confrontando i tipi azionali di portare e $\mathrm{i}$ loro traducenti, osserviamo che la relazione di traduzione risulta produttiva 
per ogni tipo di azione e che lo stesso tipo di azione, malgrado gli oggetti diversi, viene realizzato in ognuna delle serie ${ }^{7}$ :

1) Fabio porta il bagaglio / la borsa / la busta. Fabio nosi prtljag / torbu / kesu.

2) Fabio porta i fiori / i cioccolatini / il dolce a Maria. Fabio donosi cveće / čokoladice / slatkiš Mariji.

3) Fabio porta i fiori / il cibo / i bicchieri al tavolo. Fabio donosi cveće / hranu / čaše do stola.

4) Marta porta con sé la borsa / il cellulare / i documenti. Marta uzima torbu / mobilni telefon / dokumente.

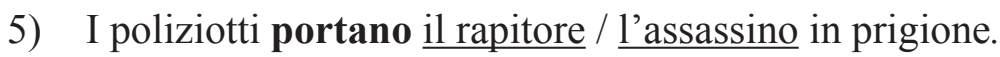
Policajci vode otmičara / ubicu u zatvor.

6) Fabio porta la motocicletta / la bicicletta in garage. Fabio uteruje motor / bicikl u garažu.

7) L'autotrasportatore porta la merce / i mobili con il camion. Prevoznik prevozi robu / nameštaj kamionom.

8) Il nastro trasportatore porta i pacchi / i biscotti. Prenosna traka prenosi pakete / keks.

La sistematicità delle traduzioni all'interno del tipo conferma che i tipi azionali possono essere considerati a un livello ontologico indipendente dal linguaggio (Moneglia 2011: 3). In base all'analisi delle variazioni primarie di 'portare' e dei traducenti serbi, viene confermata l'affermazione di Moneglia e Panunzi (Moneglia \& Panunzi 2010: 38) che non c'è una relazione di traduzione uno a uno tra verbi generali di azione in quanto $i$ verbi generali sono polisemici.

\section{CONCLUSIONE}

Come è stato mostrato, il verbo serbo nositi e i suoi prefissati non si estendono su tutti i tipi azionali di 'portare'. Infatti, Ježek (2011: 24) nota che "attraverso il lessico le lingue "ritagliano" in modo diverso gli stessi concetti". Come è stato evidenziato, da IMAGACT risulta che 'portare' è traducibile anche con i verbi generali serbi voditi e voziti e i loro prefissati. Casadei (2014: 66) afferma che "ogni parola inserita in un atto linguistico concreto instaura una relazione con le altre parole presenti in esso", ma in

\footnotetext{
${ }^{7}$ Gli esempi (1-8) sono nostri.
} 
diverse lingue la relazione stabilita da un elemento lessicale non deve presentarsi nello stesso modo visto che in ogni lingua ogni parola si presenta con la propria rete e la propria struttura (Lo Cascio, 2012: 15). Infatti, in base all'analisi delle variazioni di 'portare' e i suoi traducenti serbi in IMAGACT, siamo propensi a concludere che in serbo risultano rilevanti le caratteristiche dell'oggetto tematico. Da IMAGACT emerge che, a causa delle restrizioni relative al peso dell'oggetto tematico, nositi non si può applicare ai campi in cui 'portare' è equivalente a 'parcheggiare'. Inoltre, dagli esempi esaminati risulta che il verbo generale voditi (e i suoi prefissati) non si estendono ai tipi azionali riguardanti gli oggetti inanimati.

Infine, segnaliamo che IMAGACT può essere uno strumento utile per l'acquisizione di italiano L2. Per esempio, se l'apprendente serbo volesse verificare come in IMAGACT viene tradotto in serbo il verbo 'portare', può usare Dictionary la funzione di IMAGACT nella quale è possibile guardare la scene rappresentanti i tipi azionali. Cliccando sull'icona indicante la lingua serba, si possono vedere i lemmi serbi attribuiti al tipo azionale selezionato. Inoltre, utilizzando la funzione Compare l'utente può verificare i tipi azionali nei quali 'portare' corrisponde a un equivalente serbo prescelto.

Come è stato osservato, IMAGACT permette l'analisi dei tipi azionali in diverse lingue. Perciò, l'analisi di altri verbi generali in italiano e i loro traducenti serbi potrebbero rappresentare un interessante argomento di una futura ricerca contrastiva tra le due lingue.

\section{BIBLIOGRAFIA}

Baker, C. F. et al. (1998). The Berkeley Framenet Project. In Proceedings of the 17th International Conference on Computational Lingustics COLING '98 (Vol. 1, pp. 86-90). Stroudsburg: Association for Computational Linguistics.

Belić, A. (2000). Nauka o građenju reči. Glagoli. Glagolski vid. Beograd: Zavod za udžbenike i nastavna sredstva.

Bertinetti, P. M. (2011). Il verbo. In L. Renzi et al. (a cura di), Grande grammatica italiana di consultazione (Vol. 2, pp. 131-161). Bologna: Il Mulino.

Casadei, F. (2014). Lessico e semantica. Roma: Carocci.

D'Achille, P. (2003). L'italiano contemporaneo. Bologna: Il Mulino.

Fellbaum, C. (1998). WordNet: An Electronic Lexical Database. Cambridge: MIT Press.

Ferrari, A. \& Zampese, L. (2016). Grammatica: parole, frasi, testi dell'italiano. Roma: Carocci. 
Gagliardi, G. (2014). Validazione dell'ontologia dell'azione IMAGACT per lo studio e la diagnosi del Mild Cognitive Impairment. Testo disponibile al sito: http://www.gloriagagliardi.com/wp-content/uploads/2013/12/ Tesi_Gloria_color.pdf.

Graffi, G. (2012). La frase: l'analisi logica. Roma: Carocci.

Ježek, E. (2011). Lessico. Bologna: Il Mulino.

Kipper Schuler, K. (2005). Verbnet: A broad-coverage, comprehensive verb lexicon. Testo disponibile al sito: http://verbs.colorado.edu/ kipper/Papers/dissertation.pdf.

Klajn, I. (2007). Grammatica della lingua serba. Beograd: Zavod za udžbenike i nastavna sredstva.

Lentovskaya, A. (2007). Una nuova possibile classificazione azionale dei verbi russi. In I. Ricci \& C. Bertini (a cura di), Quaderni del Laboratorio di Linguistica della Scuola Normale Superiore di Pisa. Testo disponibile al sito: http://linguistica.sns.it/QLL/QLL07_08/Anna_Lentoskaya_tot.pdf.

Lepschy, G. C. (2007). Parole, parole, parole e altri saggi di linguistica. Bologna: Il Mulino.

Lo Cascio, V. (2012). Nelle reti del lessico. In S. Ferreri (a cura di), Lessico e lessicologia (pp. 3-27). Roma: Bulzoni.

Moneglia, M. (2005). Mettere. La semantica empirica del verbo di azione più frequente nel lessico verbale italiano. In M. Biffi, O. Calabrese \& L. Salibra (a cura di), Italia linguistica: discorsi di scritto e di parlato. Nuovi studi di linguistica italiana per Giovanni Nencioni (pp. 261-282). Testo disponibile al sito: http://lablita.dit.unifi.it/preprint/preprint-moneglia nencioni.pdf.

Moneglia, M. (2010). Predicati generali ad alta frequenza nei corpora orali delle lingue romanze. In M. Iliescu, H. Siller-Runggaldier \& P. Danler (a cura di), Actes du XXVe CILPR (Vol. 4, pp. 497-506). Testo disponibile al sito: http://lablita.dit.unifi.it/preprint/preprint.2008-0206.6774236130 .

Moneglia, M. (2012). Un caso di Annotazione Semantica del Parlato con Claire Blanche- Benveniste. In R. Druetta (a cura di), Claire BlancheBenveniste. La linguistique à l'école de l'oral (Vol. 1, pp. 133-148). Testo disponibile al sito: http://lablita.dit.unifi.it/preprint/moneglia_ claire2011.

Moneglia, M. \& Panunzi, A. (2010). I verbi generali nel corpora del parlato. Un progetto di annotazione semantica cross-linguistica. In I. Korzen \& E. Cresti (a cura di), Language, Cognition and Identity. Extension of the Endocentric/EsocentricTypology (pp. 27-46). Testo disponibile al sito: http://lablita.dit.unifi.it/preprint/Fup2010_. 
Moneglia, M. et al. (2012). La variazione dei verbi generali nei corpora di parlato spontaneo. L'ontologia IMAGACT. In H. Mello, M. Pettorino $\&$ T. Raso (a cura di), Proceedings of the VIIth GSCP International Conference (pp. 406-411). Testo disponibile al sito: http://lablita.dit. unifi.it/preprint/imagact gscp2012.

Mrazović, P. (2009). Gramatika srpskog jezika za strance. Novi Sad: Izdavačka knjižarnica Zorana Stojanovića, Sremski Karlovci.

Panunzi, A. \& Moneglia, M. (2003). La variazione semantica del verbo nel lessico dei corpora di LABLITA. In F. Albano Leoni et al. (a cura di), Il parlato italiano, Atti del Convegno Nazionale di Napoli, 13-15 febbraio, 2003. Testo disponibile al sito: http://lablita.dit.unifi.it/preprint/Fup2010_MonegliaPanunzi.

Piper, P. \& Klajn, I. (2013). Normativna gramatika srpskog jezika. Novi Sad: Matica srpska.

Salvi, G. (2013). Le parti del discorso. Roma: Carocci.

Stakić, M. (2010). Morfo(no)loške teme. Beograd: Društvo za srpski jezik i književnost.

Stanojčić, Ž. (2010). Gramatika srpskog književnog jezika. Beograd: Kreativni centar.

Strudsholm, E. (2011). Verbi di movimento. In Enciclopedia dell'italiano Treccani. Testo disponibile al sito: http://www.treccani.it/enciclopedia/ verbi-di-movimento_(Enciclopedia_dell\%27Italiano)/.

Tomelleri, V. S. (2008). L'aspetto verbale serbo tra tipologia e diacronia. In A. Alberti et al. (a cura di) Contributi italiani al XIV Congresso Internazionale degli Slavisti (pp. 11-61). Firenze: Firenze University Press.

Wittgenstein, L. (1953). Philosophical Investigations. Oxford: Blackwell. Yi, P. (2015). Verbi di azione in italiano e in cinese mandarino. Testo disponibile al sito: https://flore.unifi.it/retrieve/handle/2158/1040257/132642/ Tesi-PanYi.pdf.

\section{THE PRIMARY VARIATION OF PORTARE AND ITS SERBIAN EQUIVALENTS} IN IMAGACT

Summary

The most frequent verbs in all spoken languages are action verbs denoting physical actions performed by the agent (Mrazović 2009: 69). According to Yi (2015: 8), many action verbs are general verbs characterized by a high level of ambiguity, not fully mutually translatable between different languages. Therefore, finding an adequate action verb in a foreign language is often problematic. In this paper, we focus on the Italian verb portare 
('carry'), aiming to contribute to research on the semantics of portare and its equivalents in the Serbian language by presenting results derived from IMAGACT, a cross-language ontology of action verbs represented in animated scenes. In the contrastive analysis of the different action types of the verb portare and its Serbian equivalents in IMAGACT, we pay special attention to the differences between the two languages, which are also due to the grammaticalized verbal aspect of Serbian.

Keywords: action verbs, action types, IMAGACT, Italian, Serbian, semantics. 